\title{
A PROSPECTIVE STUDY ON EARLY ENTERAL NUTRITION FOLLOWING GASTROINTESTINAL SURGERY
}

\section{General Surgery \\ Dr. Kaushik Associate Professor, Department of General Surgery, Burdwan Medical College And Mandal Hospital}

\section{Dr Madhusudan} Chattopadhyay*

\section{Dr. Debarshi Jana}

Assistant Professor, Department of General Surgery, Burdwan Medical College And Hospital. *Corresponding Author

Young Scientist (DST) Institute Of Post-graduate Medical Education And Research, A.J.C. Bose Road, Kolkata, West Bengal, India

\section{ABSTRACT}

BACKGROUND: The word anastomosis originates from the Greek word meaning communicating opening. Gut anastomosis is one of the frequently performed surgeries. As conventional practice following gut anastomosis, patients are kept "NIL BY MOUTH" till bowel sounds return. During this time period, patient remains with nasogastric tube for decompression of stomach and providing rest to the gut. Aim of the study on early enteral nutrition following gastrointestinal surgery.METHODS: A prospective study was carried out over a period of 18 months in dept of General Surgery BMCH Burdwan. The aim of this study was to determine whether early enteral feeding (within 48hrs) after gastrointestinal surgery is tolerable and beneficial to the patient or not. RESULTS: OF 54, 34 were male, 20 were female with a percentage of $63 \%$ and $37 \%$. Every patient were given early enteral nutrition and only 6(11.1\%) developed paralytic ileus, $16(29.6 \%)$ patients developed gastrointestinal disturbances. Among 29 who underwent anastomosis 3(10.3\%) patients had anastomotic leak. Among 54, 9(16.7\%) developed SSI (11.1\%) developed chest infection. CONCLUSION: It can be concluded that early enteral feeding induces quicker recovery of postoperative gastrointestinal movements in patients. Early enteral feeding reduces SSI $>$ It is safe and effective I postoperative patients, even if there is apparent ileus. There is no significant correlation between early enteral nutrition and development of complications (ie anastomostic leak, paralytic ileus and postoperative chest infections).

\section{KEYWORDS}

Anastomosis, Enteral Feeding, Bowel Sounds.

\section{INTRODUCTION}

The word anastomosis originates from the Greek word meaning communicating opening. Gut anastomosis is one of the frequently performed surgeries in both emergency and elective setup. Anastomosis following gut resections in emergency set up is mostly done due to traumatic rupture, benign or malignant perforation or obstruction and in certain other inflammatory conditions. Anastomosis is also done in some elective conditions like mostly due to malignancy of GI system. As conventional practice following gut anastomosis, patients are kept "NIL BY MOUTH" till bowel sounds return. During this time period, patient remains with nasogastric tube for decompression of stomach and providing rest to the gut.

Recently great emphasis has been paid on early enteral feeding within 6 to 24 hours after operation. However, in post-operative period, sometimes nutrition of the patient is maintained by TPN. Except being of high cost, TPN has its own complications like infection, metabolic disturbances and immunological disturbances. So, studies are now being conducted regarding feasibility of early enteral feeding.

Aim of the study on early enteral nutrition following gastrointestinal surgery.

\section{METHODS}

A prospective Randomized Single blind study was carried out over a period of 18 months (December 2017 to May 2019) on patients undergoing gastrointestinal surgery as laparotomy and entero-enteric anastomosis or stoma formation in operations conducted under Department of General Surgery of Burdwan Medical College and Hospital, Burdwan.

A total of 54 patients after applying inclusion and exclusion criteria were studied who underwent gastrointestinal surgery as entero-enteric anastomosis or stoma formation following laparotomy / elective closure of previously conducted ileostomies / colostomies in operations conducted under Department of General Surgery of, Burdwan Medical College and Hospital, Burdwan.

For statistical analysis data were entered into a Microsoft excel spread sheet. Data have been summarized as mean and standard deviation for numerical variables and count and percentages for categorical variables. The median and the interquartile range have been stated for numerical variables that are not normally distributed. Outcome of analysis has been compared with other established researches.

\section{RESULTS AND DISCUSSION}

Patient age ranged from (12-70) years with a mean age of $42.056 \pm$ 13.705 years. The study groups have male predominance of 34 patients or $63 \%$.

Delaney et $\mathrm{al}^{1}$, in his study found that early post-operative feeding was particularly beneficial for patients younger than 70 years old.

Petrelli et al ${ }^{2}$ reported that $73 \%$ of his patients tolerated early oral feeding without sequelae. He also showed that being male had no effect on the tolerability of early oral feeding but that the estimated amount of blood loss had a role in intolerance of early feeding; this may be related to the volume required to replenish the blood loss leading to an increase in third space fluid content which in turn may lead to bowel wall edema and prolongation of ileus.

Most common anastomotic procedure done was ileostomy closure (6 cases). Previously, ileostomies were done primarily after illeal perforation, mainly in blunt abdominal trauma patients which were common in young aged male patients. Other indications were strangulated hernia, gangrenous bowel following small bowel obstruction and protective diversion ileostomy for LAR.

Other procedures in the study were Right hemicolectomy (4 cases), Hartmann reversal (3 cases), Resection and anastomosis of small bowel ( 6 cases), of which 2 cases were jejuno-jejunal anastomosis and 4 cases were illeo-illeal anastomosis, LAR (2 cases), one Left hemicolectomy, Extended Right hemicolectomy (2 cases).

In stoma procedures 7 cases were loop ileostomy. Among them 3 were due to blunt trauma abdomen and 2 were due to strangulated hernia. Loop colostomy (6 cases) was performed to relieve acute large gut obstruction. Other stoma procedure was End colostomy (2 cases), Illeostomy and mucus fistula (4 cases), End colostomy and mucus fistula ( 3 cases) and End colostomy and Hartman (3 cases).

Out of the 54 patients 17 patients were taken as cancer surgery. 6 type of cancer surgeries were performed in the study group in $17(31.5 \%)$ patients. Loop Colostomy was performed in $6(11.1 \%)$ patients to 
relieve acute obstruction as emergency procedure. 11 (88.9\%) patients had curative resection and anastomosis for malignancies.

Reversal procedures (Hartman reversal and Colostomy reversal) which were previously indicated as a part of cancer operation ( i.e. Hartmann procedure or Loop colostomy procedure in a patient with colon cancer) were not included in the 'cancer surgery' arm in this study.

Among the 29 anastomotic procedures $4(13.8 \%)$ cases were done with stapler ( 2 cases of Right hemicolectomy, 1 case of Left hemicolectomy and 1 case of Extended right hemicolectomy).

Rest 25 (86.2\%) cases were hand sewn anastomosis done with 3/0 PDS in single layer interrupted extra-mucosal fashion.

Cornelius S Carr et al ${ }^{3}$ found that early enteral feeding [EEF] is safe, well tolerated, prevents an increase in mucosal gut permeability and is associated with a positive nitrogen balance compared to negative nitrogen balance in intravenous fluids group.

Fujii et al ${ }^{4}$ suggested that very early feeding on POD1 after colorectal resection is safe and feasible and that induced a quicker recovery of postoperative gastrointestinal movement in patients.

Difronzo et al ${ }^{5}$ prospectively analysed 200 patients during a 5- year period and demonstrated that $>80 \%$ of patients tolerated early oral feeding after colonic surgery. In that series, multivariate analysis showed that being male and undergoing a total colectomy were associated with early oral feeding intolerance; this could have been caused by an increased oral intake by male patients or prolonged ileus from retroperitoneal dissection after abdominal colectomy.

In his study by Stewart et al ${ }^{6}$, full diet was resumed within 2-13 days (mean 5 days) in EOF group and 5-14 days (mean 8 days) in DOF group which is comparable with present study.

The average time of first audible bowel sound was $2.648 \pm 1.067$ days. Bowel sounds appeared on POD 2 and POD 3 in 23 patients (42.6\%) and 11 patients $(20.4 \%)$ respectively. And bowel sound appeared in POD 4 and POD 5 in $9(16.7 \%)$ and $2(3.7 \%)$ respectively. So, predominant of patient $(63 \%)$ had early appearance of bowel sound following early enteral nutrition.

In a study by Fanaie et $\mathrm{al}^{7}$, there was no significant difference in the appearance of bowel sounds among patients of two groups $(0.5 \pm 0.6 \mathrm{vs}$ $0.5 \pm 0.5)$ days $(\mathrm{p}=0.65)$.

The average time of passage of flatus was $3.319 \pm 1.146$ days. Flatus first passed on POD 2 and POD 3 in 15 patients (27.8\%) and 14 patients (26\%) respectively. And flatus first passed on POD 4 and POD 5 in 13 $(24 \%)$ and $8(14.8 \%)$ respectively. So, predominant of patient $(63 \%)$ had early passage of flatus following early enteral nutrition.

The average time of passage of stool was $4.093 \pm 1.186$ days. Stools first passed on POD 3 and POD 4 in 17 patients (31.5\%), 14 patients $(29.6 \%)$ respectively.

And stool first passed on POD 5 and POD 6 in 13 (24\%) and 4 (7.4\%) respectively. So, predominant of patient $(61.1 \%)$ had early passage of stool following early enteral nutrition.

Sanjay Marwa et al ${ }^{8}$ demonstrated that there was significant difference in complication rates among the early fed and the delayed feeding group in patients undergoing elective intestinal anastomosis.

Di Fronzo et al ${ }^{9}$ in a study on EEF after colonic resection and anastomosis had a zero rate of anastomotic leak compared with a leak rate of $3-10 \%$ in colorectal surgery.

Ahmad et $\mathrm{al}^{10}$ showed anastomotic leak in one patient (3.57\%) in early group as compared to nine patients $(32.1 \%)$ in delayed oral feeding group after elective gut anastomosis which was statistically significant $(\mathrm{p}=0.012)$.

Paralytic postoperative ileus was defined as that form of ileus lasting more than 3 days after surgery.

Out of 54 patients 6 patients (11.1\%) developed paralytic ileus during the study. All the patients were managed conservatively. No prokinetic agents were used.

Wound infection (SSI) is common complication after resection \& anastomosis of bowel, but its incidence has decreased after invention of novel antibiotics. 9 patients $(16.7 \%)$ had purulent discharge from the surgical site and fever suggestive of SSI. All cases with discharging wound healed with conservative treatment and daily dressing. Data of this study was suggestive that early enteral feeding decreases wound infection rate significantly.

Schroeder et al ${ }^{11}$ showed that early enteral feeding post-operative is associated with better wound healing.

Sanjay Marwa et al ${ }^{8}$ in a study on early feeding after intestinal anastomosis found a significant difference in wound infection rate of $4 \%$ in study and $20 \%$ in the control group. The results of meta-analysis of 11 studies by Lewis et $\mathrm{al}^{12}$ have also shown that incidence of wound infection is $3-30 \%$ in early fed group.

A total of $6(11.1 \%)$ patients have developed postoperative chest infections during the study. All the patients were managed conservatively with antibiotics, oxygenation and incentive spirometry. Preoperative respiratory status was optimal in all patients undergoing this study.

Moore et al ${ }^{13}$ proved that early EEF reduces septic morbidity after trauma, the study group (EEF) had infection rate of $9 \%$ compared to $29 \%$ in control group.

Beier and Holgerson et al ${ }^{14}$ demonstrated a higher incidence of infection in the delayed feeding group after major abdominal surgery, $46 \%$ compared to $6 \%$ in the study group and concluded that early enteral nutrition given to patients after major abdominal surgery results in an important reduction in infectious complications.

In our study, the incidence of postoperative GI disturbances is 16 patients (29.6\%). They showed complain of nausea, vomiting, abdominal distension and diarrhoea in post-operative period. These minor gastrointestinal upsets were successfully managed with temporary conservative management which included short term restriction of oral intake, antiemetic and antibiotics.

Lewis et al ${ }^{12}$ showed that the only significant disadvantage of early enteral feeding was an increased risk of nausea and vomiting.

Chatterjee Set al ${ }^{15}$ has showed that in early feeding group the rate of nausea and vomiting, wound infection and dehiscence, respiratory tract infection, anastomotic leakage and mortality was more than delayed oral feeding group.

The mean days of postoperative stay in the hospital were $7.96 \pm 1.96$ in the study population. Only $9(16.67 \%)$ patients who had lengthy hospital stay of more than 10 days. Rest $45(83.33 \%)$ patient stayed less than 10 days in post-operative period.

Study by Stewart et $\mathrm{al}^{6}$ on EEF on colorectal resection showed that the study group had a shorter duration of hospital stay.

One patient in the study group died because of sepsis. The patient underwent Extended right hemicolectomy and for Carcinoma colon involving distal transverse colon. After a brief phase of minor gastrointestinal disturbances (nausea, vomiting), the patient developed adynamic ileus. Oral feeding according was started on POD 5. The patient was observed to have developed frank anastomotic leak on POD 7 as suggested by fever, tachycardia, and disappearance of interperistaltic sounds and appearance of feculent material in both the drain bag and the surgical site. The patient was conservatively managed with restriction of oral intake, intravenous fluids, nasogastric tube insertion, broad spectrum antibiotics, total parenteral nutrition and local wound care for the next 7 days. On POD 15, patient was re-explored and end ileostomy with mucus fistula was done. Patient developed paralytic ileus, surgical site infection and chest infection in the subsequent week after which she was shifted to intensive care unit. Mechanical ventilation was required. The patient died due to sepsis on POD 27. 
Villalba et al. ${ }^{16}$ reported that many prospective randomized studies show that early feeding decreases postoperative ileus duration and hospital stay without increasing morbidity or mortality. Early feeding also reduces all risks of infection, as well as any anastomotic risk.

\section{CONCLUSION}

Intestinal anastomosis and stoma are very frequently performed surgery as emergency and elective procedures due to traumatic rupture, benign or malignant condition or obstruction and in some other inflammatory conditions.

I. In this study, we found that bowel sounds, flatus and stools appeared in a significant shortened period of time after early enteral nutrition as compared traditional delayed oral feeding. Hence, it can be concluded that early enteral feeding induces a quicker recovery of postoperative gastrointestinal movement in patients.

II. There was a significant decrease in the incidence of surgical site infections following early enteral nutrition. So, early enteral feeding reduces postoperative wound infections.

III. However, the incidence of minor gastrointestinal disturbances (i.e. nausea, vomiting, diarrhea, distension) was significant. Nearly $30 \%$ of the patients developed gastrointestinal disturbances in the post-operative period. But almost $70 \%$ patient did not develop any significant GI disturbances. So, early enteral nutrition is generally safe and effective in postoperative patients, even if there is apparent ileus.

IV. The overall postoperative length of stay was significantly less following early enteral nutrition. Hence, early enteral feeding shortens length of hospital stay.

$\mathrm{V}$. There is no significant correlation between early enteral nutrition and development of complications (i.e. anastomotic leak, paralytic ileus and postoperative chest infections)

DISTRIBUTION OF BMI, OPERATIVE PROCEDURE OF CANCER SURGERIES, TYPE OF ANASTOMOSIS AND POSTOPERATIVE DURATION OF HOSPITALSTAY

\begin{tabular}{|c|c|c|c|}
\hline & & \begin{tabular}{|c} 
NO. OF \\
PERSON
\end{tabular} & PERCENTAGE \\
\hline \multirow[t]{4}{*}{ BMI } & $<18.5$ & 7 & $13 \%$ \\
\hline & $18.5-24.9$ & 41 & $76 \%$ \\
\hline & $25-29.9$ & 6 & $11 \%$ \\
\hline & TOTAL & 54 & $100 \%$ \\
\hline \multirow{7}{*}{\begin{tabular}{|} 
OPERATIVE \\
PROCEDURE( \\
CANCER \\
SURGERIES)
\end{tabular}} & Right Hemicolectomy & 4 & $7.4 \%$ \\
\hline & $\begin{array}{l}\text { Extended Right } \\
\text { Hemicolectomy }\end{array}$ & 2 & $3.7 \%$ \\
\hline & Left Hemicolectomy & 1 & $1.9 \%$ \\
\hline & $\begin{array}{l}\text { Low Anterior } \\
\text { Ressection }\end{array}$ & 2 & $3.7 \%$ \\
\hline & $\begin{array}{c}\text { Abdomino-perineal } \\
\text { Ressection }\end{array}$ & 2 & $3.7 \%$ \\
\hline & Loop Colostomy & 6 & $11.1 \%$ \\
\hline & TOTAL & 17 & $31.5 \%$ \\
\hline \multirow{3}{*}{\begin{tabular}{|c|} 
ANASTOMOS \\
IS
\end{tabular}} & SUTURED & 25 & $86.2 \%$ \\
\hline & STAPLED & 4 & $13.8 \%$ \\
\hline & TOTAL & 29 & $100 \%$ \\
\hline \multirow{7}{*}{\begin{tabular}{|c|} 
LENGTH OF \\
HOSPITAL \\
STAY [ DAYS \\
(POD) ]
\end{tabular}} & 6 & 12 & $22.22 \%$ \\
\hline & 7 & 15 & $27.78 \%$ \\
\hline & 8 & 9 & $16.67 \%$ \\
\hline & 9 & 6 & $11.11 \%$ \\
\hline & 10 & 3 & $5.56 \%$ \\
\hline & $>10$ & 9 & $16.67 \%$ \\
\hline & TOTAL & 54 & $100 \%$ \\
\hline
\end{tabular}

REFERENCE

1. Delaney CP, Fazio VW, Senagor AJ, Robinson B et al. Fast track post-operative management protocol for patients with high comorbidity undergoing complex abdominal and pelvic colorectal surgery. Br J Surg 2001;88:1533-38.

2. Petrelli NJ, Cheng C, Driscoll D, Miguel A et al. Early post-operative oral feeding after colectomy: an analysis of factors that may predict failure. Ann Surg Onco 2001;8:796800.

3. Carr C.S, Ling KDE, Boulos P, Singer M. Randomised trial of safety and efficacy of immediate postoperative enteral feeding in patients undergoing gastrointestinal resection BMJ 1996; $312 \cdot 869-871$

4. Takaaki Fujii, Hiroki Morita, Toshinaga Sutoh, Reina Yajima, Satoru Yamaguchi, Soichi Tsutsumi, Takayuki Asao, and Hiroyuki Kuwano . Benefit of Oral Feeding as Early as One Day After Elective Surgery for Colorectal Cancer: Oral Feeding on First Versus Second Postoperative Day. Int Surg. 2014 May-Jun; 99(3): 211-215.

5. Difronzo A, Yamin N, Patel K. Benefits of early feeding and early hospital discharge in elderly patients undergoing open colon resection. J Am Coll Surg. 2003;197(5):747-52.

6. Stewart BT, Woods RJ, Collopy BT, Fink RJ, Mackay JR, Keck JO . Early feeding after elective open colorectal resection: a prospective randomized control trial. Australian New Zealnd Journal of surgery 1998; Feb;68 (2): 125-128.

7. Fanaie S A, Ziaee SA. Safety of early oral feeding after gastrointestinal tract anastomosis. Indian Journal of Surgery. 2005; 67: 185-18

8. Sanjay Marwa, Rajesh Godara, Rahul Goyal, NishaMarwah, R. Karwasra. Early enteral nutrition following gastrointestinal anastomosis. Internet journal of gastroenterology $2008 ; 7$ number 1

9. L. Andrew DiFronzo, Judith Cymerman, Theodore X. Oconnell . Factors affecting early postoperative feeding following elective open colon resection. Archive of surgery 1999 134: $941-946$

10. Halim A, Ahmad K, Ahmad I. Comparative effectiveness of early vs delayed ora feeding after elective intestinal anastomosis. Ophthalmology Update. 2014;75-8.

11. Ahmet et al. A randomized controlled trial evaluating early versus traditional oral feeding after colorectal surgery. Clinics (Sao Paolo). 2011 Dec; v66(12).

12. Lewis SJ, Egger M, Sylvester PA. Early enteral feeding versus "nil by mouth" after gastrointestinal surgery: systemic review and meta-analysis of controlled trials. BMJ. 2001;323:773-6

13. EE Moore, Moore FA. Immediate enteral nutrition following multi-system trauma Journal of American college of nutrition 1991; 10:633-648

14. R Beier-Holgersen and S Boesby. Influence of postoperative enteral nutrition on postsurgical infections. Gut. 1996 Dec; 39(6): 833-835.

15. Chatterjee S, Bala SK, Chakraborty P, Dey R, Sinha S, Ray R, et al. A comparative study between early enteral feeding (within 24 hours) versus conventional enteral feeding after enteric anastomosis. Bangladesh J Med Sci. 2012:11(04):273-83.

16. Villalba Ferrer F, Bruna Esteban M, Garcia Coret MJ, Gacia Romero J, Roig Vila V. Evidence of early oral feeding in colorectal surgery. Rev Esp Enferm Dig (Mad)2007;99(12):709-13. 